\title{
Evaluation of the Effect of (S)-3,4- Dicarboxyphenylglycine as a Metabotropic Glutamate Receptors Subtype 8 Agonist on Thermal Nociception Following Central Neuropathic Pain
}

\author{
Marjan Hosseini, Mohsen Parviz, Alireza P. Shabanzadeh, Elham Zamani \\ Department of Physiology, School of Medicine, Tehran University of Medical Sciences, Tehran, Iran
}

Study Design: In this study, we decided to change the activity of periaqueductal gray (PAG)'s metabotropic glutamate receptors subtype 8 (mGluR8) by means of its specific agonist, (S)-3,4-dicarboxyphenylglycine (DCPG), and by knock downing it with mGluR8 siRNA. We then evaluated the changes in animal pain threshold levels in the face of painful thermal stimuli (thermal hyperalgesia).

Purpose: Although several mechanisms have been examined for central neuropathic pain, researchers have so far failed to find the precise mechanism for the development and progression of this type of pain. Hyperalgesia is one of the most important complications of central neuropathic pain and there is not a consensus among researchers about the exact cause of this complication. In this study, we investigated the effect of activation of the PAG region mGluR8 on the threshold of pain response to thermal noxious stimulus in rats and measured mGluR8 expression.

Overview of Literature: Spinal cord injury (SCI) produces an decrease in mGluR2/3 expression in the injured and vehicle-treated groups compared to normal levels, APDC and L-AP4 treated groups had higher expression levels of mGluR2/3. These findings suggesting that the level of mGluR expression after SCI may modulate nociceptive responses.

Methods: Male Wistar rats were randomly assigned to five groups ( $n=10$ per group). The clip compression injury model was used to induce chronic central neuropathic pain. Three weeks after SCI, DCPG, siRNA, or normal saline were administered to the intra-ventrolateral PAG region. Withdrawal threshold to the noxious thermal stimulus (e.g., heat hyperalgesia) was assessed through the tail-flick test. In order to assure involvement of this receptor, pain responses were compared with mice that received GRM8 siRNA.

Results: We found that the mGluR8 agonist DCPG increased lead to an increased expression of mGluR8 in the PAG region. We also found that SCI can decrease the threshold of response to painful thermal stimuli; however, activation of mGluR8 with DCPG agonist did not significantly improve the tail-flick response.

Conclusions: The results revealed that activation of mGluR8 in PAG is not capable of improving the thermal hyperalgesia threshold. Based on the decreased expression of mGluR8 after SCl induced by clip compression injury and its significant increase after treatment of siRNA against mGluR8, this method might still hold promise as an effective treatment of neuropathic pain. It can be concluded that increased expression of mGluR8 is due to the fact that DCPG prevents the death of neurons that express these receptors.

Keywords: Hyperalgesia; Metabotropic glutamate receptor 8; Spinal cord injuries; 3,4-Dicarboxyphenylglycine; Periaqueductal gray; Neuralgia

Received Dec 3, 2019; Revised Jan 19, 2020; Accepted Feb 18, 2020

Corresponding author: Mohsen Parviz

Department of Physiology, Tehran University of Medical Sciences, Poursina Street, Tehran, Iran

Tel: +98-21-66419484, Fax: +98-66419484, E-mail: parvizmo@tums.ac.ir 


\section{Introduction}

Damage to the central nervous system usually results in oversensitivity to both painful and non-painful environmental stimuli. Increased susceptibility to stimuli is known as hyperalgesia. Neuropathic pain following spinal cord injury (SCI) is common and hyperalgesia is a serious complication that can arise from that initial injury. Several mechanisms have been defined for hyperalgesia that occurs after central neuropathic pain $[1,2]$.

Neuropathic pain is a form of chronic pain that is defined as pain caused by injury or disease of the sensoryphysical system and is manifests as allodynia, hyperalgesia, and spontaneous pain. In addition to altering the nervous system, neuropathic pain reduces the level of daily physical activity. This pain may directly and/or indirectly affect the structure and function of nerve cells $[3,4]$. The prevalence of Iranians suffering from neuropathic pain is calculated between $2 \%-40 \%$ of adults and is it is increasing over time [5]. About 20\% (50 million) of the American population suffers from neuropathic pain, highlighting the importance of this disease [6]. SCI and the neuropathic pain that follows can lead to numerous complications including, vertebral ulcer, rupture of the blood-brain barrier, vascular disruption, axonal degeneration, induction of apoptosis and cellular loss, up- or downregulation of neurotransmitters and their receptors, and neuronal excitotoxicity and hyperactivity $[7,8]$. The two main subcategories of pain are nociceptive and neuropathic pain. Nociceptive pain is acute and it usually has a specific cause, but neuropathic pain results from damage to the central or peripheral nervous system and hyperalgesia is a form of neuropathic pain born out of that damage [9].

In fact, many hypothesize that the phenomenon of central sensitization is the main causational mechanism of hyperalgesia. In central sensitization, nociceptive neurons in the dorsal horns become sensitized by peripheral tissue damage or inflammation [10]. In other words, central tenderness occurs due to persistent and prolonged stimulation of pain (i.e., chronic pain). Research has shown that repeated exposure to painful stimuli alters the pain response threshold and will in fact be stronger in the face of painful stimuli [10]. Glutamate is one of the most important neurotransmitters responsible for central sensitization at the spinal cord level. Glutamate exerts its functions through two completely different receptor types: ion-channel receptors (inotropic) and metabotropic glutamate receptors (mGluRs). There are eight different types of mGluRs (mGluR1, mGluR2, etc.) that are divided into three groups. Group I include mGluR1 and mGluR5 and their action can be excitatory, increase glutamatedependent transfers, and have can activate phospholipase C. Group II include mGluR2 and mGluR3, and group III include mGluR4, 6, 7, and 8. Groups II and III are presynaptic and have a suppressive and autoreceptive role in glutamate-related transmissions due to their association with inhibitory G-Pro [11]. It seems that controls applied from supraspinal levels to the posterior horn may also be effective in hyperalgesia, via reduced descending inhibition or via enhanced descending facilitation. Therefore, it is expected that glutamate partially suppresses hypersensitivity in the posterior horn of the spinal cord and also prevents the transmission of exaggerated pain messages by activating group III mGluRs (mGluRIII) in the periaqueductal gray (PAG) region, from which the descending inhibition begins. PAG activation, either through electrical stimulus or via agonists, can lead to significant analgesic responses [12]. Therefore, in this study we altered the activity of PAG mGluR8 by means of its specific agonist, (S)-3,4-dicarboxyphenylglycine (DCPG), as well as inhibiting its activity with siRNA mGluR8 knockdown and evaluated changes in animal pain threshold levels in response to painful thermal hyperalgesia stimuli. Also, considering the role of mGluRIII in modifying pain messages, we examined the changes in PAG's mGluR8 expression after SCI and injection with specific mGluR8 siRNA, followed by treatment with mGluR8 agonist DCPG.

\section{Materials and Methods}

\section{Animals}

Male Wistar rats, weighing 200-250 g, were received from the experimental animal's laboratory of Tehran University of Medical Sciences and kept for at least 1 week in special cages before being tested for environmental compatibility. Animals had unlimited access to food and water, were without dietary restrictions, and were kept under controlled laboratory conditions (temperature: $21^{\circ} \mathrm{C} \pm 1^{\circ} \mathrm{C} ; 12$ hours light/dark cycle). This study was approved by the Experimental Animal's Laboratory of Tehran University of Medical Sciences (Institutional Animal Care approval no., IR.TUMS.MEDICINE.REC.1396.2229). 


\section{Experimental design}

Animals were randomly divided into five groups ( $\mathrm{n}=10$ per group): Sham, SCI, Vehicle, siRNA, and DCPG. In the sham group, the spinal cord of rats was exposed and closed without any intervention and injury. In the SCI group, animals received SCI without any treatment. In the vehicle group, animals received single injection of normal saline. Animals in the siRNA group received single injection of mGluR8 specific siRNA, GRM8 siRNA. In the treatment (DCPG) group, animals received single injection of DCPG as a mGluR8 agonist. Animals in all groups, except the sham group, underwent SCI surgery.

\section{Induction of chronic central neuropathic pain}

The clips compression injury model was used to induce chronic central neuropathic pain [13]. Briefly, after anesthesia (ketamine $80 \mathrm{mg} / \mathrm{kg}$ and xylazine $10 \mathrm{mg} / \mathrm{kg}$ ), the animals' hair was shaved from the back and laminectomy was performed to expose the spinal cord on the thoracic region of T6-T8. A micro-vascular clip (Harvard Apparatus, Holliston, MA, USA) was placed vertically on the exposed thoracic spinal cord for 60 seconds. Following spinal compression, the clip was removed and then the muscles and skin were closed with $4 / 0$ silk.

\section{Drugs and tracer administration}

To perform direct intra-ventrolateral PAG administrations of the drugs (DCPG, siRNA and normal saline), 3 weeks after SCI, animals were anesthetized with ketamine $(80 \mathrm{mg} / \mathrm{kg})$ and xylazine $(10 \mathrm{mg} / \mathrm{kg})$. A stainless steel 22-gauge guide cannula was stereotaxically lowered towards the intra-ventrolateral PAG $(30 \mathrm{nmol} / \mathrm{rat}$ DCPG $\left(\mathrm{EC}_{50}=30 \mathrm{nmol}\right)$ and normal saline, volumes of $200 \mathrm{~nL}$ drug solutions). PAG coordinates applied according to "Atlas of Paxinos (1986)" were (A: -7.8 mm and L: 0.5 $\mathrm{mm}$ from bregma, V: $4.3 \mathrm{~mm}$ below the dura) [14]. Other surgical procedures and the form of drug injection were consistent with the previous study described by Marabese et al. [14].

\section{5. mGluR8 siRNA treatment after spinal cord injury}

In order to determine the role of mGluR8 in symptoms improvement of SCI, we used GRM8 siRNA (specific
siRNA for mGluR8) as a knockdown model. Afterwards, the animals' symptoms were evaluated following SCI in the absence of mGluR8 receptors and compared the results with agonist group. The siRNA used in this study was purchased from MyBioSource Inc. (MBS8233032; MyBioSource Inc., San Diego, CA, USA) with the following sequence:

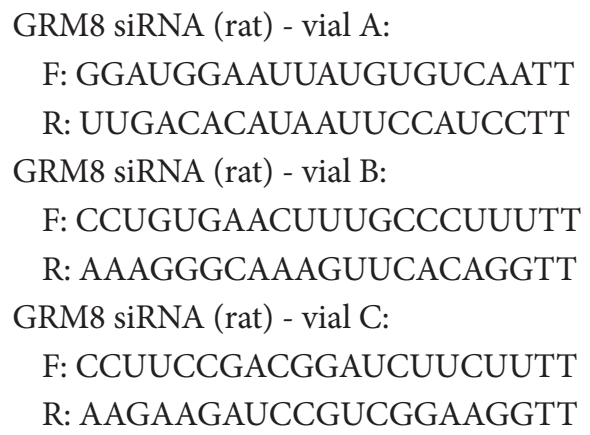

Final solution was prepared by combining vials A, B, and $C$ with a 1:1:1 ratio. Three weeks after SCI, the animals were injected with $5 \mu \mathrm{L}$ siRNA solution in the intraventrolateral PAG (total volume of $5 \mu \mathrm{L}$ ).

\section{Behavioral study: thermal hyperalgesia}

The level of nociception activity after SCI was evaluated through the tail-flick test [15]. Rats were placed on the device and were allowed to adjust to the new environment. When the exploratory behavior ceased, an infrared source producing a calibrated heating beam (diameter, $1 \mathrm{~mm}$ ) was placed under the tail base and triggered together with a timer. The time for the first movement of the tail was noted. Each measurement was repeated three times, with at least a 4-minute interval [16].

\section{Histological studies: Western blot}

Brain tissues (three rats from each group) were homogenized in $200 \mu \mathrm{L}$ ice-cold lysis buffer with an electric homogenizer. The blade was rinsed twice with another 200 $\mu \mathrm{L}$ lysis buffer, and then centrifuged at $13,000 \times \mathrm{g}$ at $4^{\circ} \mathrm{C}$ for 20 minutes. Supernatants were removed and stored at $-80^{\circ} \mathrm{C}$ until use. To investigate protein expression, 2.5 $\mu \mathrm{L}$ of lysate was removed to perform a protein quantification assay. Using a plate reader, the protein concentration of $150 \mu \mathrm{g}$ was determined for each cell lysate. Changes in protein expression of PAG's mGluR8 (anti-MGLUR8, 
orb161742) were assessed through Western blotting according to the blotting protocol [17].

\section{Statistical analysis}

Two-way analysis of variance and Bonferroni post hoc tests were applied for the time course (comparison of expression before versus after treatment). In all calculations, a $p$-value $<0.05$ was considered to be significant.

\section{Results}

\section{Tail-flick test}

Thermal hyperalgesia was measured by the latency of tail withdrawal response to noxious heat stimulation. As indicated in Fig. 1, sham group animals without SCI the pain response threshold is quite normal and exhibited no changes pre- or post-treatment. However, in other groups with SCI, the threshold of response to painful thermal stimuli was significantly lower than that of sham group ( $\mathrm{F}$ $[10,108]=21.7 ; p<0.001)$. This is indicative of the impact of SCI on neuropathic pain and its subsequent complications. Intra-ventrolateral PAG microinjection of DCPG (30 $\mathrm{nmol} / \mathrm{rat}$ ) did not change the tail withdrawal latency when measured 30 and 60 minutes after treatment compared with pre-treatment responses. Additionally, no significant difference between the DCPG, the SCI, or siRNA groups were observed $(p<0.05)$ (Fig. 1).

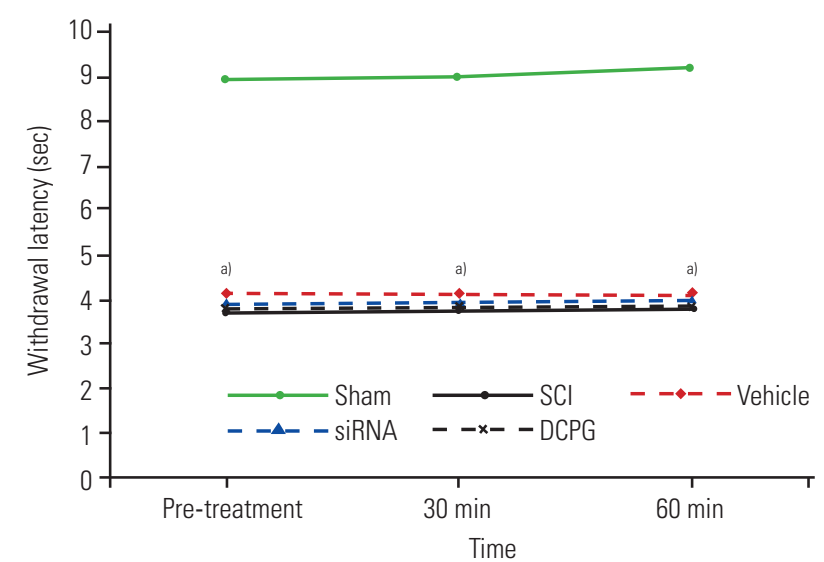

Fig. 1. The effect of DCPG on tail withdrawal threshold after thermal (heat) stimulus. The mean withdrawal threshold was not changed significantly after administration of DCPG compared to the SCI and siRNA groups. SCl, spinal cord

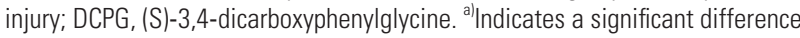
between all intervention groups and the sham group at the level of 0.001 .

\section{Expression of mGluR8}

The evaluation of expression of mGluR8/GAPDH density ratios via Western blot analysis of the PAG area showed that SCI significantly reduced the expression of mGluR8 compared to the sham group (Fig. 2A) $(p<0.001)$. Conversely, administration of DCPG significantly increased expression of mGluR8 in PAG area relative to the SCI and vehicle groups. Finally, administration of siRNA significantly reduced expression of the expression of these receptors $(p<0.001)$ (Fig. $2 \mathrm{~B})$.

\section{Discussion}

Spinal tissue injury is associated with sensitization of nociceptors and subsequent changes in the excitability of spinal cord neurons in dorsal horn is termed central sensitization. This phenomenon is considered to be the primary underlying cause in the development of hyperalgesia because central sensitization is considered to reflect plasticity at spinal synapses, the descending inhibition and

(A)
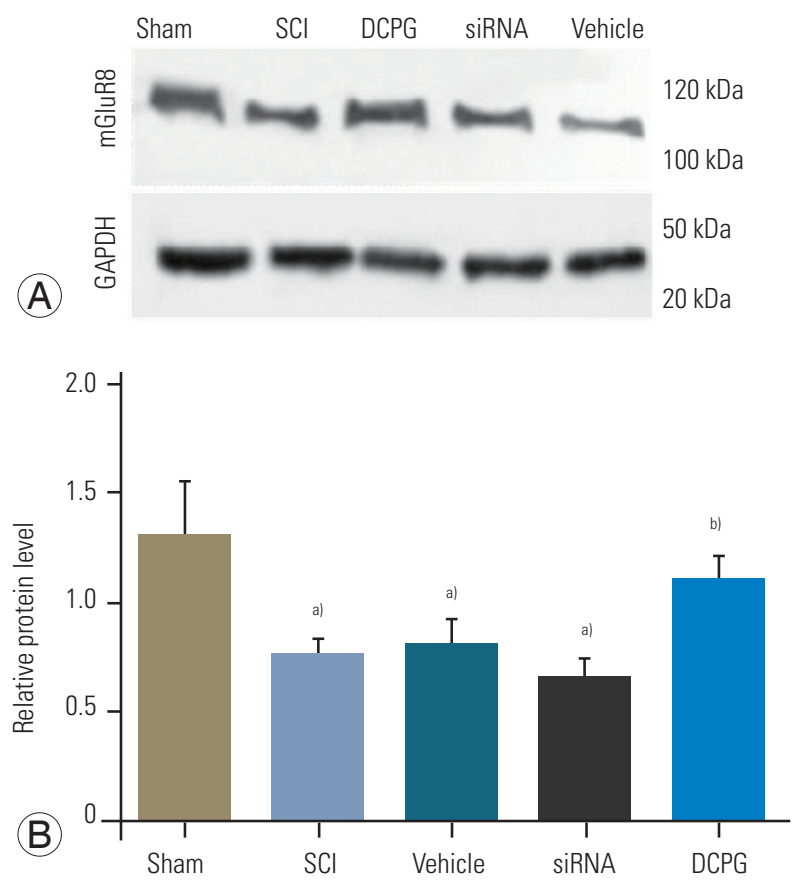

Fig. 2. Determination of periaqueductal gray mGluR8 expression by Western blot immunoassay (A). As shown in the diagram (B), after injury, the expression level of mGluR8 decreased significantly compared to the sham group. DCPG treatment slightly changed the amount of these receptors in comparison with the siRNA group ( $\mathrm{n}=3$ independent rats/condition). SCI, spinal cord injury; DCPG, (S)-3,4-dicarboxyphenylglycine; mGluR8, metabotropic glutamate receptors subtype $8 .{ }^{a}$ IIndicates a significant difference at $p<0.001$ in comparison to the sham group. ${ }^{b}$ Indicates a significant difference at $p<0.001$ in comparison to the $\mathrm{SCl}$ group. 
facilitation (all of them cross the spinal cord) [10]. In our study, we examined the effect of DCPG, a selective agonist of mGluR8, on pain response and thermal hyperalgesia following SCI. The results indicate that single injection of DCPG with a dose of $30 \mathrm{nmol}$ per rat in the intra-ventrolateral PAG does not reduce thermal hyperalgesia, but does have a significant effect on the expression mGluR8 in the PAG region, which has been shown to play a role in pain suppression [12].

The PAG region is located within the tegmentum of the midbrain and it projects to many sites in the brain stem and spinal cord and also receives many inputs from the spinal cord and the peripheral nervous system. The ascending pain fibers and temperature send information to the PAG via the spinomesencephalic tract. In PAG, there are many glutamatergic, GABAergic and opioidergic neurons that play an important role in the process of pain suppression in the form of a descending antinociceptive pathway.

Descending inhibitory and facilitatory pathways that originate from or lead to the PAG, effect pain messages at many sites in the brainstem and spinal cord [18]. In this study, we injected an agonist of mGluR8, DCPG, into the PAG to assess its effect on initiation and amplification descending inhibitory pathways as well as assessing the pain threshold against noxious thermal stimuli (thermal hyperalgesia) in rats with SCI.

Any stimuli, such as mechanical, chemical, or thermal, that leads to tissue damage is classified as noxious stimuli. This process eventually leads to perception of pain at the brain level, termed nociception. If these stimuli cause damage to the peripheral or central nerves or the nociceptors, hyperalgesia will occur. Hyperalgesia is divided into two types: primary hyperalgesia is contributed to sensitization of peripheral nerve (this is known as peripheral sensitization) and send exaggerated pain messages to higher centers, whereas secondary hyperalgesia is due to changes in the synaptic communication of spinal cord and higher brain areas (central sensitization). We focused our study on secondary hyperalgesia or central sensitization.

In general, metabotropic glutamate receptors, can exhibit quite unexpected behavior, depending on their location or the conditions. However, the most well-known mechanism for hyperalgesia is central hypersensitivity and therefore a hyperactivity of glutamate N-methyl-Daspartate (NMDA) receptors in the spinal cord. During the development and progression of hyperalgesia, syn- apses created by pain-transmitting fibers will become a long-term potentiation model in the spinal cord, according to a pattern that occurs during inflammation. In these synapses, glutamate mediates resonance of pain via NMDA receptors [19]. In our study, the use of DCPG at a dose of $30 \mathrm{nmol}$ failed to significantly increase the threshold response to painful thermal stimuli in SCI animals nor sham group. Therefore, it can be concluded that the use of DCPG, at least at this dose, did not have an effect on spinal cord NMDA receptor activity. Therefore, we can conclude that it also had no significant effect on facilitated stimulatory synapses of nociceptors with PAG downstream fibers. We believe that the fact that mGluR8 agonist treatment had no effect on animal pain thresholds against painful thermal stimuli is that, activation of mGluR8 in the PAG region and initiation of the antinociceptive pathway to the posterior horn alone cannot address NMDA overactivation in dorsal horn. In our previous study, we demonstrated that the only way to deal with the heat hyperalgesia is to focus on glutamate NMDA receptors at the dorsal horn surface and focus on other pain-relieving techniques that can improve the other complications of neuropathic pain, which have distinct mechanisms [13]. However, neuropathic pain has many side effects, one of which is thermal hyperalgesia. Future research should focus on examining the impact of treatments on all aspects of this type of pain.

SCI usually initiates many biochemical processes. Among these changes, the increase or decrease of various receptors that are involved in the process of suppressing or promoting pain, including mGluR8, are effected and can alter the pain message [20]. The subtypes of mGluRs have different spatial and temporal expression patterns and there is a basic need for further study on each of the eight members of this receptor family.

In a 2015 study on mGluR4, from the mGluRIII family, it was shown that the use of this receptor's agonist in culture medium of neuronal progenitor stem cells from different parts of the rat brain can reduce cell death compared to the mGluR4 siRNA group. Therefore, the increase observed in expression of PAG mGluR8 is likely due to a decrease in the mortality of cells containing these receptors in the PAG [21]. It can be concluded that increased expression of mGluR8 observed in our study is due to the fact that DCPG prevents the death of neurons that express these receptors. Since the decreased expression of mGluR8 is parallel to development of thermal 
hyperalgesia following SCI (in SCI and siRNA group), understanding the expression change of mGluR8 after SCI and treatment may give insight into mechanisms underlying the development of chronic central pain. Additionally, we found that following SCI, mGluR8 expression was significantly reduced in the SCI group when compared to the sham group. Paralleling these results, it was observed that the animals' threshold to a painful thermal stimulus (tail-flick test) in the SCI and also siRNA groups was significantly reduced compared to the sham group, indicating that mGluR8 activity is effective in suppressing the pain message. On the other hand, we observed that after treatment with DCPG, the expression of mGluR8 significantly increased compared to the SCI and siRNA groups. According to other studies, DCPG has been shown to reduce cellular apoptosis, we have found that the death rate of nerve cells expressing this receptor has decreased and we saw an increase in the expression of mGluR8. We expected that the animal pain threshold would be higher following an increase in mGluR8 expression, due to the suppressive role of these receptors on pain, but we did not observe a significant change in animal pain threshold for thermal hyperalgesia. A search of the literature revealed that the most common cause of thermal hyperalgesia is an increase in glutamate current via NMDA receptors.

\section{Conclusions}

Neuropathic pain has many complications, but hyperalgesia and allodynia are the most important and debilitating side effects of this kind of pain. Furthermore, hyperalgesia and allodynia also have different sub-types. To date, no effective treatments have been found for these complications. For this reason, pain researchers are trying to find a way to alleviate these complications that involve targeting different receptors and neurotransmitters. It is true that the DCPG administration did not alter the pain threshold of animals in the tail-flick test or in thermal hyperalgesia, but this does not mean that this treatment will not have any effect on pain relief. Most likely, for treatment of heat hyperalgesia using intrathecal injections or other therapeutic doses of DCPG may be useful; focusing on another mechanisms like spinal NMDA receptors may also provide a therapeutic benefit. These results could guide future research to finding a direct way to treat neuropathic pain complications and reduce the number of trials and errors in future studies. Central neuropathic pain has many as- pects and all of these aspects must be taken into account when deciding on a treatment. Due to the decreased expression of mGluR8 after the SCI and its significant increase after treatment, especially compared to the siRNA group, this method can be effective in the treatment of pain. Our research team will continue to study issues related to DCPG administration, such as allodynia and mechanical hyperalgesia, and we have some interesting results that will be published at a later date.

\section{Conflict of Interest}

No potential conflict of interest relevant to this article was reported.

\section{Acknowledgments}

The authors appreciate the insightful cooperation of the Animal Laboratory of Tehran University of Medical Sciences. This experiment was conducted in the Department of Physiology. This study was supported by the grant research funds of the School of Medicine, Tehran University of Medical Sciences.

\section{Author Contributions}

Study design: MP, APS; data collection: MH; and literature search: EZ

\section{References}

1. Merskey HE. Classification of chronic pain: descriptions of chronic pain syndromes and definitions of pain terms. Pain 1986;Suppl 3:226.

2. Bonica JJ. Evolution and current status of pain programs. J Pharm Care Pain Symptom Control 1993;1:31-44.

3. Devor M. Nerve pathophysiology and mechanisms of pain in causalgia. J Auton Nerv Syst 1983;7:371-84.

4. Munglani R, Hunt SP. Molecular biology of pain. Br J Anaesth 1995;75:186-92.

5. Mohammadzadeh F, Faghihzadeh S, Asadi Lari M, et al. A fairly comprehensive survey of chronic pain in Iranian population: prevalence, risk factors, and impact on daily life. Health Scope 2015;4:e25467.

6. Dahlhamer J, Lucas J, Zelaya C, et al. Prevalence of chronic pain and high-impact chronic pain among 
adults: United States, 2016. MMWR Morb Mortal Wkly Rep 2018;67:1001-6.

7. Kakulas BA. A review of the neuropathology of human spinal cord injury with emphasis on special features. J Spinal Cord Med 1999;22:119-24.

8. Norenberg MD, Smith J, Marcillo A. The pathology of human spinal cord injury: defining the problems. J Neurotrauma 2004;21:429-40.

9. Treede RD. Chapter 1 pain and hyperalgesia: definitions and theories. Handb Clin Neurol 2006;81:3-10.

10. Latremoliere A, Woolf CJ. Central sensitization: a generator of pain hypersensitivity by central neural plasticity. J Pain 2009;10:895-926.

11. Bleakman D, Alt A, Nisenbaum ES. Glutamate receptors and pain. Semin Cell Dev Biol 2006;17:592-604.

12. Marabese I, de Novellis V, Palazzo E, et al. Effects of (S)-3,4-DCPG, an mGlu8 receptor agonist, on inflammatory and neuropathic pain in mice. Neuropharmacology 2007;52:253-62.

13. Hosseini M, Karami Z, Janzadenh A, et al. The effect of intrathecal administration of muscimol on modulation of neuropathic pain symptoms resulting from spinal cord injury: an experimental study. Emerg (Tehran) 2014;2:151-7.

14. Paxinos G, Watson C. The rat brain in stereotaxic coordinates: the new coronal set. 5th ed. Boston: Elsevier Academic Press; 2006.
15. Marabese I, Rossi F, Palazzo E, et al. Periaqueductal gray metabotropic glutamate receptor subtype 7 and 8 mediate opposite effects on amino acid release, rostral ventromedial medulla cell activities, and thermal nociception. J Neurophysiol 2007;98:43-53.

16. D'Amour FE, Smith DL. A method for determining loss of pain sensation. J Pharmacol Exp Ther 1941;72:74-9.

17. Merkler D, Metz GA, Raineteau O, Dietz V, Schwab ME, Fouad K. Locomotor recovery in spinal cordinjured rats treated with an antibody neutralizing the myelin-associated neurite growth inhibitor Nogo-A. J Neurosci 2001;21:3665-73.

18. Kurien BT, Scofield RH. Western blotting: an introduction. Methods Mol Biol 2015;1312:17-30.

19. Vanegas H, Schaible HG. Descending control of persistent pain: inhibitory or facilitatory? Brain Res Brain Res Rev 2004;46:295-309.

20. Sandkuhler J. Models and mechanisms of hyperalgesia and allodynia. Physiol Rev 2009;89:707-58.

21. Mills CD, Fullwood SD, Hulsebosch CE. Changes in metabotropic glutamate receptor expression following spinal cord injury. Exp Neurol 2001;170:244-57.

22. Zhang Z, Ma W, Wang L, et al. Activation of type 4 metabotropic glutamate receptor attenuates oxidative stress-induced death of neural stem cells with inhibition of JNK and p38 MAPK signaling. Stem Cells Dev 2015;24:2709-22. 